\title{
Wideband compressed sensing for Cognitive Radios using optimum detector with no reconstruction
}

\author{
Jonathan Verlant-Chenet André Bourdoux Jean-Michel Dricot Philippe De Doncker François Horlin \\ Université Libre de Bruxelles - OPERA Dpt., Avenue F.D. Roosevelt 50, B-1050 Brussels, Belgium \\ jverlant@ulb.ac.be
}

\begin{abstract}
Cognitive radios need devices capable of sensing a large range of frequencies in order to detect the presence of primary networks and reuse their bands when they are not occupied. Due to the large spectrum to be sensed and the high power signal dynamics, low-cost implementation of the analog front-ends leads to imperfections. In this paper, we solve this problem with compressed sensing. The introduced maximum likelihood method is computationally simple since it does not require any signal reconstruction, unlike most methods in the current literature. Moreover, the metric is optimum, works for any modulation scheme and is independent of the emitted signal knowledge. The results are supported with Matlab simulations, a statistical study is performed and the probability of error is plotted for different cases, proving the efficiency of the estimator in a range of plausible SNRs and subsampling factors.
\end{abstract}

\section{INTRODUCTION}

Cognitive radios are devices aware of the spectrum availability that surrounds them, in order to reuse the frequency bands allocated to a primary user when they are free. First, they must analyze a certain range of frequencies in order to discover potential opportunities (that is, free frequency bands). Then, the devices communicate in the licensed bands, while avoiding interference with the primary networks.

A standard terminal consists of an analog front-end (for continuous signal processing such as frequency conversion, filtering and amplification) and a digital processor (allowing demodulation and synchronization algorithms). The design of the analog front-end is critical in the case of cognitive radios since they have to work on a large spectrum composed of several bands, and therefore use more complex front-ends. Nevertheless, it is possible to analyze a large number of frequency bands with a sampling rate that does not need the Nyquist criterion; it is called compressed sensing. If a signal is uniformly undersampled (that is, at a constant sampling frequency below Nyquist rate), replicas appear and signal reconstruction is possible under certain constraints [1]. In this paper, a random non-uniform subsampling that avoids replicas is considered.

The compressed sensing is widely used for applications such as signal acquisition, compression, medical imaging and sensor networks, but most of the time, authors propose reconstruction algorithms which involve computational complexity typically polynomial in the signal length. For example, reconstruction in [2] and [3] is performed by solving $\ell_{1}$-norm optimization using linear programming techniques such as Basis Pursuit (BP) or iterative greedy algorithms such as Orthogonal Matching Pursuit (OMP). Authors in [4] use wavelet transforms as a recovery tool, and [5] implements an Interior-Point Method. However, inference tasks such as detection, classification and estimation do not require the reconstruction of the signal. In this case, only the estimation of a relevant sufficient statistic is needed [6].
Techniques for compressed sensing with no reconstruction have recently appeared. The authors of [6] use a NeymanPearson detector where a likelihood ratio $\frac{f_{1}(r)}{f_{0}(r)}\left(f_{1}\right.$ and $f_{0}$ being respectively the PDF in case of presence and absence of a primary user in the subsampled signal $r$ ) is compared to a certain threshold chosen so that the probability of false alarm is equal to a fixed value. Unfortunately, the test statistic still depends on the emitted signal. In [7], their metric is the cosine of the angle between the recieved vector $r$ and columns of a matrix $\Theta$ (defined such as $r=\Theta s=\Phi \Psi s$ where $\mathrm{s}$ is the frequency domain transmitted signal, $\Phi$ is a subsampling matrix and $\Psi$ is an IFFT matrix). When there is only one frequency band in use, since $r$ is obtained by multiplying the nonzero member of $s$ by its corresponding column in $\Theta$, it is possible to determine the occupied frequency by maximizing the cosine of the angle between $r$ and the columns of $\Theta$. Although this method is then generalized to the case of $\mathrm{M}$ occupied frequency bands, it is not optimal and does not minimizes the impact of the noise. [8] introduces a tone detector using the idea of a peak excision by frequency-domain peak search. The strongest peaks (detected in the received subsampled signal spectrum) are removed, one after the other, while the remaining leakage they introduced is kept minimized (using least-squares minimization). The algorithm is optimum but, in our case, we need to be able to detect modulated signals.

This paper takes it one step further in the direction of cognitive radios and it introduces a simple frequency estimator based on the Maximum Likelihood approach and independent of the transmitted symbols. Thus, the detector does not need any information (symbols, modulation scheme, etc.) about the primary user, which is common for cognitive radio scenarios. Also, since it maximizes a likelihood function, it is optimal. Moreover, it works for any type of modulated signals except real constellations like BPSK.

The paper is organized as follows. We first introduce a new system model. Then, we compute the likelihood function. An analytical study of this function is performed and the results are supported with Matlab simulations.

\section{SYSTEM MODEL}

\section{A. General case system model}

Fig. 1 illustrates the overall system when a linearly modulated communication signal is present at $\mathrm{M}$ center frequencies $f_{m}$.

At each transmitter, independent QAM modulated symbols $I_{k, m}$ of variance $\sigma_{I}^{2}$ are transmitted at the rate $1 / T_{\text {symb. The }}$ symbols are supposed independent and identically distributed 


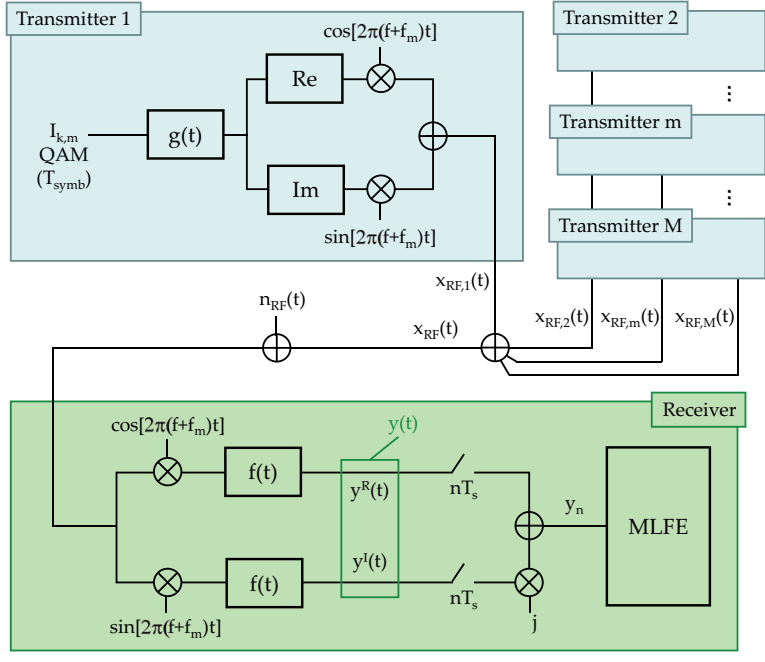

Fig. 1. Bandpass architecture with $\mathrm{M}$ transmitters.

(IID), with null mean and no correlation between real and imaginary parts. Note that the detector metric computed in section III will be independent of the transmitted symbols (and therefore also of the modulation scheme). The sequence is low-pass filtered by $g(t)$ in order to remove the out-of-band components (typically a Square Root Raised Cosine filter is used), and then modulated on frequency $f_{m}$. The resulting baseband signal, for all transmitters, is given by:

$$
x(t)=\sum_{m=1}^{M} \sum_{k} I_{k, m} g\left(t-k T_{\mathrm{symb}}\right) e^{j 2 \pi f_{m} t}
$$

The radio-frequency signal $x_{\mathrm{RF}}(t)$ is obtained after upconversion to the carrier frequency $f$ and transmitted through the channel, where additive white Gaussian noise $n_{\mathrm{RF}}(t)$, of one-sided power spectral density (PSD) equal to $\frac{N_{0}}{2}$, corrupts the received signal.

At the receiver, the RF signal is down-converted to the baseband domain for complex operation and low-pass filtered by $f(t)$ in order to limit the observation to the desired bandwidth $B_{f}$. As we assume an AWGN channel with no multipath, the baseband received signal $r(t)$ can be expressed as:

$$
r(t)=x(t)+n(t)
$$

where $n(t)$ is the baseband equivalent noise of variance $\sigma_{n}^{2}=N_{0} B_{f}$. After sampling at the rate $1 / T_{\mathrm{s}}$, the obtained received sequence $y_{n}$ is used for the spectrum sensing (here called MLFE for Maximum Likelihood Frequency Estimator, see section III).

\section{B. Scenario under consideration}

In our scenario, we assume the presence of one user and will try to find the frequency band that it uses. For instance, a user is occupying one of the ten $10 \mathrm{MHz}$ channels available in a range of $100 \mathrm{MHz}$. This scenario involves subsampling at rates that do not respect the Nyquist criterion (i.e. inferior to $100 \mathrm{MHz}$ ), such as $20 \mathrm{MHz}$ or $10 \mathrm{MHz}$ (respectively a subsampling by a factor 5 or 10).
Since only one frequency band of center frequency $f_{B}$ is used, the expression (1) of the baseband transmitted signal contains one center frequency $f_{m}=f_{B}$ and has a variance equal to $\sigma_{x}^{2}=\sigma_{I}^{2} \int_{-\infty}^{\infty}|g(t)|^{2} d t$.

The received signal is subsampled at $\mathrm{N}$ random instants $t_{n}$, with a mean rate $1 / T_{\text {sub }}$, smaller then the Nyquist rate. The obtained received sequence is

$$
r_{n}=r\left(t_{n}\right)=\sum_{k} I_{k} g\left(t_{n}-k T_{\mathrm{symb}}\right) e^{j 2 \pi f_{B} t_{n}}+n\left(t_{n}\right)
$$

This sequence can be expressed as a matricial product:

$$
\begin{aligned}
\underline{r} & =\underline{\underline{S}} \cdot(\underline{x}+\underline{n})=\underline{\underline{S}} \cdot(\underline{\underline{E}} \cdot \underline{\underline{G}} \cdot \underline{I}+\underline{n}) \\
& =\underline{\underline{S}} \cdot \underline{\underline{E}} \cdot \underline{\underline{G}} \cdot \underline{I}+\underline{\underline{S}} \cdot \underline{n}
\end{aligned}
$$

where $\underline{I}$ is a vector containing the K QAM symbols kept in the window of observation and $\underline{r}$ is a vector containing $\mathrm{N}$ received samples. The K symbols are multiplied by a convolution matrix $\underline{\underline{G}}$ of size $W \times K$ (representing the convolution with the Square Root Raised Cosine filter) and a frequency shift diagonal matrix $\underline{\underline{E}}$ containing exponential factors, such as:

$$
E_{n n^{\prime}}= \begin{cases}\exp \left(j 2 \pi f_{B} t_{\mathrm{n}}\right) & \text { if } n=n^{\prime} \\ 0 & \text { else }\end{cases}
$$

$\underline{\underline{S}}$ is the subsampling rectangular matrix of dimensions $N \times W$ containing only one non-null element on each line corresponding to the random times of sampling. For instance, if $y_{n}$ is a 9 samples received sequence that we want to subsample with a factor $3, \underline{\underline{S}}$ could be

$$
\underline{\underline{S}}=\left(\begin{array}{ccccccccc}
1 & 0 & 0 & 0 & 0 & 0 & 0 & 0 & 0 \\
0 & 0 & 0 & 0 & 1 & 0 & 0 & 0 & 0 \\
0 & 0 & 0 & 0 & 0 & 0 & 1 & 0 & 0
\end{array}\right)
$$

so that the subsampled sequence $r_{n}$ only contains the first, fifth and seventh elements of $y_{n}$.

\section{MAXIMUM LIKelihood FReQUenCy Estimator (MLFE)}

Since Maximum Likelihood estimation is used to estimate parameters of a statistical model, we will use it to find the central frequency of the band in use. The criterion is to maximize the probability density function of the signal while knowing the frequency [9]:

$$
\widehat{f}=\arg \max _{f} p(\underline{r} \mid f) .
$$

According to the law of total probability, $p(X)=\int_{Y} p(X \mid Y) p(Y)$ and (6) becomes:

$$
\widehat{f}=\arg \max _{f} \int_{\underline{I}} p(\underline{r} \mid f, \underline{I}) p(\underline{I})=\arg \max _{f} \mathbb{E}_{\underline{I}}[p(\underline{r} \mid f, \underline{I})]
$$

where $\mathbb{E}_{\underline{I}}$ denotes the expectation with respect to the symbols $\underline{I}$. 


\section{A. PDF computation}

The purpose of this subsection is to compute the PDF $p(\underline{r} \mid f, \underline{I})$. In the expression (3) of the received signal, the first term is deterministic as $\underline{I}$ is supposed to be known; only the second term is random and has a Gaussian density of probability by definition of the AWGN. Thus, the PDF of $\underline{r}$ is the same as a Gaussian random variable with mean $\underline{\underline{S}} \cdot \underline{\underline{E}} \cdot \underline{\underline{G}} \cdot \underline{I}$ and variance $\sigma_{n}^{2} \underline{\underline{I}}_{N}$ (where $\underline{\underline{I}}_{N}$ denotes the identity matrix of size $\mathrm{N}$ ).

We obtain:

$$
\begin{aligned}
p(\underline{r} \mid f, \underline{I}) & =C \cdot \exp \left(-\frac{1}{2 \sigma_{n}^{2}}|\underline{r}-\underline{\underline{S}} \cdot \underline{\underline{E}} \cdot \underline{\underline{G}} \cdot \underline{\underline{I}}|^{2}\right) \\
& =C \cdot \exp \left(-\frac{1}{2 \sigma_{n}^{2}}\left(\underline{r}^{H} \cdot \underline{r}-2 \Re\left[\underline{I}^{H} \cdot \underline{z}\right]+\underline{I}^{H} \cdot \underline{\underline{M}} \cdot \underline{I}\right)\right)
\end{aligned}
$$

where

- $C=\left(2 \pi \sigma_{n}^{2}\right)^{\frac{N}{2}}$ is independent of $\underline{I}$ and $f$

- $\underline{r}^{H} \cdot \underline{r}$ is independent of $\underline{I}$ and $f$ as $\underline{r}$ is measured at the receiver - $\underline{z}=\underline{\underline{G}}^{H} \cdot \underline{\underline{E}}^{H} \cdot \underline{\underline{S}}^{H} \cdot \underline{r}$ is a matched filter operation on $\underline{r}$. It does not depend on $I$ but will affect the maximization with respect to $f$ (the frequency dependency being in the $\underline{\underline{E}}$ matrix) in the expression (7).

- $\underline{\underline{M}}=\underline{\underline{G}}^{H} \cdot \underline{\underline{E}}^{H} \cdot \underline{\underline{S}}{ }^{H} \cdot \underline{\underline{S}} \cdot \underline{\underline{E}} \cdot \underline{\underline{G}}$. It is possible to show that the product $\underline{\underline{S}} . \underline{\underline{E}}$ can permute if we remove from $\underline{\underline{E}}$ the lines that are not selected by $\underline{\underline{S}}$. Thus, we have $\underline{\underline{M}}=\underline{\underline{G}}^{H} \cdot \underline{\underline{S}}^{H} \cdot \underline{\underline{S}} \cdot \underline{\underline{G}}$ which is independent of $f$.

The PDF (8) can be simplified as:

$$
p(\underline{r} \mid f, \underline{I})=D \cdot \exp \left(-\frac{1}{2 \sigma_{n}^{2}}\left(-2 \Re\left[\underline{I}^{H} \cdot \underline{z}\right]+\underline{I}^{H} \cdot \underline{\underline{M}} \cdot \underline{I}\right)\right)
$$

where $D=C \cdot \exp \left(-\frac{1}{2 \sigma_{n}^{2}}\left(\underline{r}^{H} \cdot \underline{r}\right)\right)$ is a factor that does not affect the expectation nor the maximization with respect to $f$.

For low $S N R$, the factor $-\frac{1}{2 \sigma_{n}^{2}}$ is small and the exponential can be expanded as a Maclaurin serie limited to the second order: $\exp (\lambda) \approx 1+\lambda+\lambda^{2}$. As cognitive radios often deal with low $S N R s$, this expansion will be used in the next section.

\section{B. Likelihood function computation}

The likelihood function has the form

$$
\begin{aligned}
\max _{f} \mathbb{E}_{\underline{I}}[p(\underline{r} \mid f, \underline{I})] & =\max _{f} \mathbb{E}_{\underline{I}}[D \cdot \exp (\lambda)] \\
& \approx \max _{f} \mathbb{E}_{\underline{I}}\left[D \cdot\left(1+\lambda+\lambda^{2}\right)\right] \\
& \approx \max _{f}\left(1+\mathbb{E}_{\underline{I}}[\lambda]+\mathbb{E}_{\underline{I}}\left[\lambda^{2}\right]\right)
\end{aligned}
$$

where $\lambda=-\frac{1}{2 \sigma_{n}^{2}}\left(-2 \Re\left[\underline{I}^{H} \cdot \underline{z}\right]+\underline{I}^{H} \cdot \underline{\underline{M}} \cdot \underline{I}\right)$

The first expectation is:

$$
-2 \sigma_{n}^{2} \mathbb{E}_{\underline{I}}[\lambda]=\underline{z}^{H} \cdot \mathbb{E}_{\underline{I}}[\underline{I}]+\mathbb{E}_{\underline{I}}\left[\underline{I}^{H}\right] \cdot \underline{z}+\mathbb{E}_{\underline{I}}\left[\underline{I}^{H} \cdot \underline{\underline{M}} \cdot \underline{I}\right]
$$

The first two terms are null by hypothesis, and the third term is independent of $f$. This expectation has therefore no effect on the maximization.

The second expectation is:

$$
\begin{aligned}
4\left(\sigma_{n}^{2}\right)^{2} \mathbb{E}_{\underline{I}}\left[\lambda^{2}\right] & =\mathbb{E}_{\underline{I}}\left[\underline{I}^{H} \underline{\underline{M}} \underline{I} \underline{I}^{H} \underline{\underline{M}} \underline{I}\right] \\
& -4 \mathbb{E}_{\underline{I}}\left[\underline{I}^{H} \underline{\underline{M}} \underline{I} \Re\left[\underline{I}^{H} \underline{z}\right]\right] \\
& +\mathbb{E}_{\underline{I}}\left[\left(\underline{I}^{H} \underline{z}\right)^{2}\right] \\
& +2 \mathbb{E}_{\underline{I}}\left[\left(\underline{I}^{H} \underline{z z}^{H} \underline{I}\right)\right] \\
& +\mathbb{E}_{\underline{I}}\left[\left(\underline{z}^{H} \underline{I}\right)^{2}\right]
\end{aligned}
$$

The term (10) is independent of $f$. The terms (12) and (14) are null for the same reason:

$$
\begin{aligned}
\mathbb{E}_{\underline{I}}\left[\left(\underline{I}^{H} \underline{z}\right)^{2}\right] & =\mathbb{E}_{\underline{I}}\left[\left(\sum_{k} I_{k}^{*} z_{k}\right)^{2}\right] \\
& =\mathbb{E}_{\underline{I}}\left[\sum_{k}\left(I_{k}^{*}\right)^{2} z_{k}^{2}+2 \sum_{i} \sum_{j>i} I_{i}^{*} I_{j}^{*} z_{i} z_{j}\right] \\
& =\sum_{k} \mathbb{E}_{\underline{I}}\left[\left(I_{k}^{*}\right)^{2}\right] z_{k}^{2}+2 \sum_{i} \sum_{j>i} \mathbb{E}_{\underline{I}}\left[I_{i}^{*} I_{j}^{*}\right] z_{i} z_{j} \\
& =0
\end{aligned}
$$

because symbols are IID by hypothesis and

$$
\mathbb{E}_{\underline{I}}\left[I_{k}\right]=\mathbb{E}_{\underline{I}}\left[\left(I_{k}\right)^{2}\right]=0 .
$$

The term (11) can be developed as:

$$
\mathbb{E}_{\underline{I}}\left[\underline{I}^{H} \underline{\underline{M}} \underline{I} \Re\left[\underline{I}^{H} \underline{z}\right]\right]=\frac{\sigma_{I}^{2}}{2} \operatorname{tr}(\underline{\underline{M}})\left(\mathbb{E}_{\underline{I}}\left[\underline{I}^{H}\right] \underline{z}+\underline{z}^{H} \mathbb{E}_{\underline{I}}[\underline{I}]\right)
$$

where $\operatorname{tr}(\underline{\underline{M}})$ denotes the trace of the matrix $\underline{\underline{M}}$. Because of (15), (11) is thus also null.

The only non-zero term (11) is equal to:

$$
\begin{aligned}
\mathbb{E}_{\underline{I}}\left[\left(\underline{I}^{H} \underline{z z}^{H} \underline{I}\right)\right] & =\sum_{k} \mathbb{E}_{\underline{I}}\left[I_{k}^{*} I_{k}\right] z_{k}^{*} z_{k}+\sum_{i} \sum_{j \neq i} \mathbb{E}_{\underline{I}}\left[I_{i}^{*} I_{j}\right] z_{i} z_{j}^{*} \\
& =\sigma_{I}^{2} \sum_{k} z_{k}^{*} z_{k}=\sigma_{I}^{2}\left(\underline{z}^{H} \underline{z}\right)=\sigma_{I}^{2}|\underline{z}|^{2}
\end{aligned}
$$

The resulting expectation is:

$$
4\left(\sigma_{n}^{2}\right)^{2} \mathbb{E}_{\underline{I}}\left[\lambda^{2}\right]=2 \sigma_{I}^{2}|\underline{z}|^{2} .
$$

After removing all the terms that do not depend on $f$, the likelihood function (9) become:

$$
\max _{f}|\underline{z}|^{2}=\max _{f}\left|\underline{\underline{G}}^{H} \underline{\underline{E}}^{H} \underline{\underline{S}}^{H} \underline{r}\right|^{2}
$$

which represents the energy of the matched filtered subsampled received signal. This function does not depend on the transmitter symbols $\underline{I}$ and has a very simple form which has only one dependency on $f$ (through the $\underline{\underline{E}}$ matrix). Moreover, it can be computed as a simple dyadic product. 


\section{Simulations}

To support previous analytical results with Matlab ${ }^{\complement}$ simulations, we implemented the MLFE for the mentioned scenario. For this simulation, the estimator is computing $|z|^{2}$ based on a $3 \mu s$ observation window. This energy is computed for each carrier frequency ranging from $-50 \mathrm{MHz}$ to $-50 \mathrm{MHz}$ (i.e. $110 \mathrm{MHz}$ baseband total bandwidth) every $10 \mathrm{MHz}$. Symbols are sent in only one of these bands, which carrier frequency is: $f_{B}=20$ MHz.

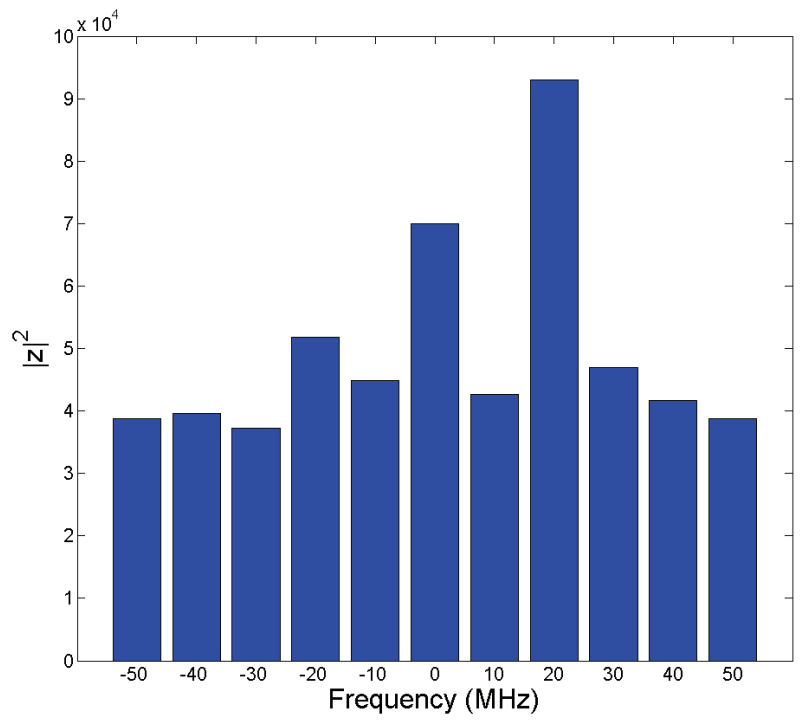

Fig. 2. Computing of $|z|^{2}$ for every carrier frequency.

Fig. 2 shows that the metric $|z|^{2}$ is maximum for $f_{B}=20$ $\mathrm{MHz}$, therefore giving the carrier frequency of the most probable frequency band in use.

Let us now use this context to perform a statistical study of our frequency estimator. For that, we execute 5000 realizations of the estimation and compute a probability of error $p_{e}$ (given the occupied frequency, $p_{e}$ is computed by dividing the number of unsuccessful detections by 5000). This probability depends on the Signal-to-Noise Ratio $S N R$ and the subsampling factor $c$ (ratio between the Nyquist minimum sampling frequency and the subsampling mean rate), which are the two parameters of our simulations.

Fig. 3 shows that the probability of error quickly rise for SNRs below a certain value. Above this value, the probability of error tends to zero and our estimator is reliable.

As expected, when the subsampling factor $c$ increases (i.e. when the mean sampling rate decreases), the estimator performance decreases and the curves are shifted to the right. On Fig. 3 are shown the probability of error for three different mean sampling rates : 50, 20, and $10 \mathrm{MHz}$.

Fig. 4 shows the performance of the estimator when the subsampling factor $c$ is varying. As expected, the probability of error increases with $c$.

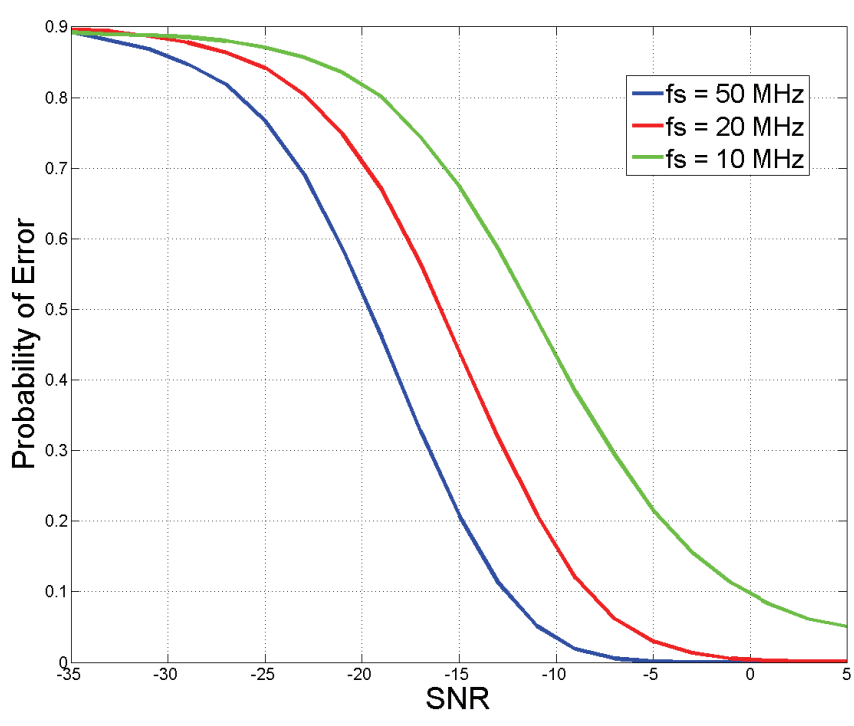

Fig. 3. Probability of error in the case of subsampling factors 2,5 and 10 (respectively corresponding to mean sampling rates 50,20 and $10 \mathrm{MHz}$ ).

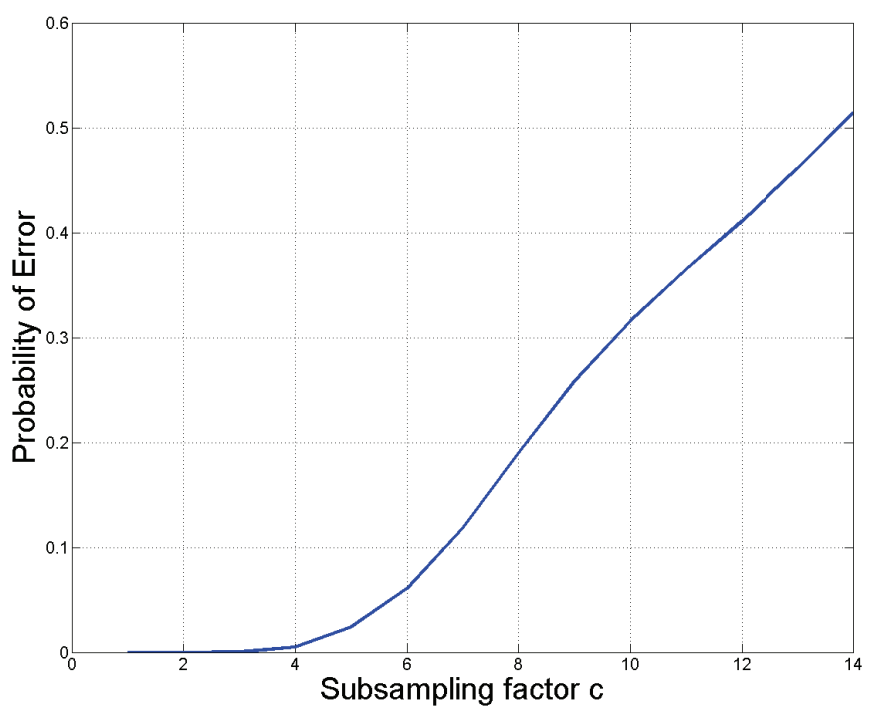

Fig. 4. Probability of error versus subsampling factor for a SNR of $-5 \mathrm{~dB}$

\section{CONCLUSION}

This paper introduces a new frequency estimator combining maximum likelihood method and subsampling. The computed metric is optimum, works for any modulation scheme and is independent of the emitted signal knowledge, which is useful for cognitive radio scenarios. A statistical study is performed and the probability of error is computed for varying SNR and subsampling factor.

As expected, the probability of error increases when the mean sampling frequency or the SNR decreases. The simulations prove the efficiency of the estimator in a range of plausible SNRs and subsampling factors, and also show the limits of it. Thus, for a given probability of error, it is possible to estimate a 
the subsampling factors that may satisfy a range of SNRs.

The results of this paper considers a scenario with one occupied frequency band. Further works on this topic will take in account a scenario where two (or more) frequency bands are occupied by users and multipath scenarios.

\section{REFERENCES}

[1] Y. L. Polo, "Compressive wideband spectrum sensing for cognitive radio applications," M.S. thesis, Delft University of Technology, 2009.

[2] Zhuizhuan Yu and S. Hoyos, "Compressive spectrum sensing front-ends for cognitive radios," in IEEE International Conference on Systems, Man and Cybernetics (SMC), 2009.

[3] Y. L. Polo, Ying Wang, A. Pandharipande, and G. Leus, "Compressive wide-band spectrum sensing," in IEEE International Conference on Acoustics, Speech and Signal Processing (ICASSP), 2009.

[4] Zhi Tian and Georgios B. Giannakis, "A wavelet approach to wideband spectrum sensing for cognitive radios," in IEEE Cognitive Radio Oriented Wireless Networks and Communications (CROWNCOM), 2006.

[5] Kim Seung-Jean, K. Koh, M. Lustig, S. Boyd, and D. Gorinevsky, "An interior-point method for large-scale 11-regularized least squares," IEEE Journal of Selected Topics in Signal Processing, vol. 1, no. 4, pp. $606-$ 617, 2007.

[6] M. A. Davenport, P. T. Boufounos, M. B. Wakin, and R.G. Baraniuk, "Signal processing with compressive measurements," IEEE Journal of Selected Topics in Signal Processing, vol. 4, no. 2, pp. 445 - 460, 2010.

[7] Jia Yuan, Pengwu Tian, and Hongyi Yu, "The detection of frequency hopping signal using compressive sensing," in International Conference on Information Engineering and Computer Science (ICIECS), 2009.

[8] A. Bourdoux, S. Pollin, A. Dejonghe, and L. Van der Perre, "Tone detection of non-uniformly undersampled signals with frequency excision," in IEEE International Conference on Acoustics, Speech and Signal Processing (ICASSP), 2011.

[9] H. Meyr, M. Moeneclaey, and S. Fechtel, Digital Communication Receivers: Synchronization, Channel Estimation, and Signal Processing, John Wiley and Sons, Inc., 1997. 